\title{
Separation of the diffuse contribution to the specular x-ray scattering of multilayer films
}

\author{
V. P. Romanov, ${ }^{1}$ S. V. Ulyanov, ${ }^{1}$ V. M. Uzdin, ${ }^{1,2}$ G. Nowak, ${ }^{3}$ K. Shokuie, ${ }^{3}$ and H. Zabel ${ }^{3}$ \\ ${ }^{1}$ St.-Petersburg State University, Ul'yanovskaya ul.1, Petrodvorets, St.-Petersburg 198904, Russia \\ ${ }^{2}$ St.-Petersburg State University of Information Technologies, Mechanics and Optics, 49, Kronverkskij, St.-Petersburg 197101, Russia \\ ${ }^{3}$ Department of Physics, Ruhr-University Bochum, D-44780 Bochum, Germany
}

(Received 2 September 2010; published 8 October 2010)

\begin{abstract}
X-ray reflectivity measurements from multilayer films contain information not only about the layer thickness and interface roughness but also about correlations of the interface roughness parallel and perpendicular to the interface. Due to the finite size of the receiving detector slit, it collects both purely specular and diffusely scattered radiation. Separation of the diffuse contribution to the spectra of specular reflectivity is an important task of processing the experimental data. For this purpose we suggest and implement a measurement method for specularly scattered scans integrated over several solid angles determined by the size of detector slits. Since in all cases the specular reflectivities coincide, this approach allows a separation of the diffuse and pure specular contributions. This separation is, in turn, required for obtaining information about the in- and out-of-plane structural correlation lengths. The method was used to analyze the experimental data of two multilayer systems with different interface properties: $\mathrm{MgO} /[\mathrm{V}(1.212 \mathrm{~nm}) / \mathrm{Fe}(0.715 \mathrm{~nm})]_{25} / \mathrm{V}(24.24 \mathrm{~nm}) / \mathrm{Pd}(5-8 \mathrm{~nm})$ and $\mathrm{SiO}_{2} / \mathrm{Si} /[\mathrm{CoFeB}(2.55 \mathrm{~nm}) / \mathrm{MgO}(1.8 \mathrm{~nm})]_{15}$.
\end{abstract}

DOI: 10.1103/PhysRevB.82.165416

PACS number(s): 61.05.cf, 61.05.cm

\section{INTRODUCTION}

Magnetic heterostructures and nanostructures have a huge impact on the development of modern science and technology. ${ }^{1-3}$ Perfection of layers and interfaces are of utmost importance for the functionality and performance of magnetoelectronic and spintronic devices. ${ }^{4}$ Theories describing spin transport and spin tunneling effects often assume perfect epitaxy and smooth interfaces. However, on the nanoscale real surfaces and interfaces are rough. Even one atomic layer high steps at interfaces can lead to dramatic changes in the interlayer exchange coupling, 5,6 the exchange bias effect, ${ }^{7}$ and the transport properties in nanosystems. ${ }^{8}$ Different types of roughness can be distinguished that change the macroscopic properties of magnetic nanosystems, such as structural roughness, interdiffusion, and magnetic roughness. Furthermore, roughness occurs on different length scales, each one requiring corresponding experimental tools for their analysis. Therefore, detailed information concerning the amplitude and the correlation of different types of roughnesses is of great interest for research and applications of magnetic nanosystems.

Experimental characterization and theoretical description of real interfaces is not an easy task and requires advanced methods in order to make progress. Most experimental methods give only indirect information about the interface roughness and their correlations in multilayer systems, such as Mössbauer spectroscopy, x-ray scattering, and neutron scattering. They need theoretical models to connect roughness parameters with experimental spectra. Often different physical sources can lead to the same features in the experimental spectra, which can cause ambiguities in the interpretation of experimental data. Thus further development of experimental methods and possibly modifications of common experimental procedures will be necessary for providing supplementary information about interface structures, which ultimately will improve the description of real systems.

One of the most widely used tool for the structural analysis of interfaces is hard x-ray scattering. The analysis of the specular and diffuse $\mathrm{x}$-ray scattering is an effective method for studying rough interfaces in multilayer systems. ${ }^{9,10}$ Usually interfaces are characterized by roughness amplitudes and by in-plane and out-of-plane correlation lengths. Specular reflectivities contain information about roughness amplitudes whereas the in-plane spatial correlations are determined by diffuse off-specular scattering. There are two main types of experiments for measuring the diffuse scattering. ${ }^{6,9}$ In the first type, referred to as off-specular scan, the incidence angle $\theta_{1}$ and reflection angle $\theta_{2}$ differ by a small constant mismatch angle. In the second case, called rocking scan, the orientation of the sample is varied for fixed directions of the incident and detected beams and the intensity of the x-ray scattering is analyzed usually in the vicinity of Bragg peaks. ${ }^{11,12}$ In both types of experiments the size of the detector slit remains constant. Actually the diffuse and the specular scattering are present in both types of experiments. The separation of these contributions is a challenging problem. ${ }^{13}$ It may, however, be solved by another type of experiment where both contributions to the scattering, specular and diffuse, are recorded in a standard $\theta_{1}=\theta_{2}$ scan but with several sizes of the detector slit. This work is devoted to a theoretical description of $\mathrm{x}$-ray scattering procedures with variable detector slits and the analysis of respective experimental data.

\section{GENERAL EQUATIONS}

We consider x-ray scattering by multilayer systems with rough interfaces. In what follows we assume that the glancing angles $\theta_{1}$ and $\theta_{2}$ for the incident and scattered rays exceed significantly the critical angle $\theta_{\mathrm{c}}$ for total external reflection. In this case the spectra may be described in a kinematic approximation when multiple reflections by interfaces in multilayer systems can be neglected. For the differential cross section of $\mathrm{x}$-ray scattering we have 
$\frac{d \sigma}{d \Omega}=\frac{k_{0}^{4}}{(2 \pi)^{2}}\left\langle\int_{V} \int_{V} d \mathbf{r}_{1} d \mathbf{r}_{2}\left[1-\varepsilon\left(\mathbf{r}_{1}\right)\right]\left[1-\varepsilon\left(\mathbf{r}_{2}\right)\right] e^{-i \mathbf{q} \cdot\left(\mathbf{r}_{1}-\mathbf{r}_{2}\right)}\right\rangle$.

Here $V$ is the scattering volume, $k_{0}=\omega / c$ is the wave number, $\omega$ is the angular frequency, $c$ is the light velocity in vacuum, $\varepsilon(\boldsymbol{r})$ is the permittivity, $\boldsymbol{q}=\boldsymbol{k}_{i}-\boldsymbol{k}_{s}$ is the scattering vector, $\boldsymbol{k}_{i}$ and $\boldsymbol{k}_{s}$ are the wave vectors of the incident and scattered waves, and brackets \langle\rangle mean the statistical or ensemble average.

For multilayers containing $N$ layers located in $(x ; y)$ plane, Eq. (1) can be written in the form ${ }^{14-17}$

$$
\frac{d \sigma}{d \Omega}=\frac{k_{0}^{4}}{(4 \pi)^{2} q_{z}^{2}} \int_{S} \int_{S} d \mathbf{r}_{(x y), 1} d \mathbf{r}_{(x y), 2} e^{-i \mathbf{q}_{(x y)} \cdot\left(\mathbf{r}_{(x y), 1}-\mathbf{r}_{(x y), 2}\right)} \sum_{n, m=0}^{N} \Delta \varepsilon_{n} \Delta \varepsilon_{m}\left\langle\exp \left\{-i q_{z}\left[z_{n}\left(\mathbf{r}_{(x y), 1}\right)-z_{m}\left(\mathbf{r}_{(x y), 2}\right)\right]\right\}\right\rangle,
$$

where $S$ is the film area and $\Delta \varepsilon_{n}=\varepsilon_{n+1}-\varepsilon_{n}$ is the permittivity jump at the $n$th interface. Equating $z=z_{n}\left(\mathbf{r}_{(x y)}\right)$ determines the position of this interface. We will consider the multilayer film with $N$ layers and $N+1$ interfaces. The interface number 0 separates the substrate and the first layer, the interface number $N$ is the boundary to the external media. In what follows we neglect the intermixing effect between neighboring layers.

It is convenient to present the function $z_{n}\left(\mathbf{r}_{(x y)}\right)$, which describes the interface position in the form $z_{n}\left(\mathbf{r}_{(x y)}\right)=z_{n}+h_{n}\left(\mathbf{r}_{(x y)}\right)$, where $z_{n}$ is the average position of the $n$th interface and $h_{n}\left(\mathbf{r}_{(x y)}\right)$ is the deviation from the average position $\left\langle h_{n}\left(\mathbf{r}_{(x y)}\right)\right\rangle=0$. For the differential cross section we have

$$
\begin{aligned}
\frac{d \sigma}{d \Omega}= & \frac{k_{0}^{4}}{(4 \pi)^{2} q_{z}^{2}} \int_{S} \int_{S} d \mathbf{r}_{(x y), 1} d \mathbf{r}_{(x y), 2} e^{-i \mathbf{q}_{(x y)} \cdot\left(\mathbf{r}_{(x y), 1}-\mathbf{r}_{(x y), 2}\right)} \times \sum_{n, m=0}^{N} \Delta \varepsilon_{n} \Delta \varepsilon_{m} \exp \left[-i q_{z}\left(z_{n}-z_{m}\right)\right]\left\{\left\langle\exp \left[-i q_{z} h_{n}\left(\mathbf{r}_{(x y), 1}\right)\right]\right\rangle\right. \\
& \left.\times\left\langle\exp \left[i q_{z} h_{m}\left(\mathbf{r}_{(x y), 2}\right)\right]\right\rangle+\left[\left\langle\exp \left[-i q_{z}\left(h_{n}\left(\mathbf{r}_{(x y), 1}\right)-h_{m}\left(\mathbf{r}_{(x y), 2}\right)\right)\right]\right\rangle-\left\langle\exp \left[-i q_{z} h_{n}\left(\mathbf{r}_{(x y), 1}\right)\right]\right\rangle\left\langle\exp \left[i q_{z} h_{m}\left(\mathbf{r}_{(x y), 2}\right)\right]\right\rangle\right]\right\} .
\end{aligned}
$$

Assuming that the interface structure is homogeneous within the interface plane, then the local average values of the physical parameters do not depend on the position in the $(x ; y)$ plane. In this case we have $\left\langle\exp \left[i q_{z} h_{n}\left(\mathbf{r}_{(x y)}\right)\right]\right\rangle=\left\langle\exp \left(i q_{z} h_{n}\right)\right\rangle$. Then Eq. (3) can be rewritten as

$$
\begin{aligned}
\frac{d \sigma}{d \Omega}= & \frac{k_{0}^{4}}{(4 \pi)^{2} q_{z}^{2}} \sum_{n, m=0}^{N} \Delta \varepsilon_{n} \Delta \varepsilon_{m} \exp \left[-i q_{z}\left(z_{n}-z_{m}\right)\right]\left\langle\exp \left[-i q_{z} h_{n}\right]\right\rangle\left\langle\exp \left[i q_{z} h_{m}\right]\right\rangle \\
& \times\left\{S(2 \pi)^{2} \delta\left(q_{x}\right) \delta\left(q_{y}\right)+\int_{S} \int_{S} d \mathbf{r}_{(x y), 1} d \mathbf{r}_{(x y), 2} e^{-i \mathbf{q}_{(x y)}\left(\mathbf{r}_{(x y), 1}-\mathbf{r}_{(x y), 2}\right)}\left[\frac{\left\langle\exp \left[-i q_{z}\left(h_{n}\left(\mathbf{r}_{(x y), 1}\right)-h_{m}\left(\mathbf{r}_{(x y), 2}\right)\right)\right]\right\rangle}{\left\langle\exp \left[-i q_{z} h_{n}\right]\right\rangle\left\langle\exp \left[i q_{z} h_{m}\right]\right\rangle}-1\right]\right\} .
\end{aligned}
$$

We consider a slit of width $a$ and length $b$. The $y$ axis is chosen to be parallel to the long side of the slit, which is perpendicular to the scattering plane. The experimental setup for specular and diffuse $\mathrm{x}$-ray scattering investigation is shown in Fig. 1. Figure 2 illustrates the corresponding scattering vectors. In this case for scattered intensity $\Delta \sigma$ into the solid angle $\mathrm{d} \Omega$ we have

$$
\Delta \sigma=\int_{\Delta \Omega}\left(\frac{d \sigma}{d \Omega}\right) d \Omega=\int_{-\Delta q_{y}}^{\Delta q_{y}} \frac{d q_{y}}{k_{0}} \int_{q_{x}-\Delta q_{x}}^{q_{x}+\Delta q_{x}} \frac{d q_{x}}{k_{0} \sin \theta_{2}}\left(\frac{d \sigma}{d \Omega}\right),
$$

where

$$
\Delta q_{x}=\frac{a \sin \theta_{2}}{2 L} k_{0}, \quad \Delta q_{y}=\frac{b}{2 L} k_{0}, \quad|\Delta \Omega|=\frac{a b}{L^{2}}=\frac{4 \Delta q_{x} \Delta q_{y}}{k_{0}^{2} \sin \theta_{2}} .
$$

Here $\theta_{2}$ is the glancing angle of the scattered beam, and $L$ is the distance between the sample and the detector. The reflectivity $R$ is determined as the ratio of scattered intensity within the solid angle $\Delta \Omega$ and the intensity of the incident radiation,

$$
R=\frac{1}{S \sin \theta_{1}} \int_{\Delta \Omega}\left(\frac{d \sigma}{d \Omega}\right) d \Omega
$$

It is convenient to express this coefficient as the sum of two terms $^{9}$

$$
R=R_{1}+R_{2}
$$

where

$$
R_{1}=\frac{k_{0}^{2}}{4 q_{z}^{2} \sin \theta_{1} \sin \theta_{2}} \Theta\left(\Delta q_{x}-q_{x}\right) \Theta\left(\Delta q_{x}+q_{x}\right) \sum_{n, m=0}^{N} F_{n, m},
$$




$$
R_{2}=\frac{k_{0}^{2}}{(2 \pi)^{2} q_{z}^{2} \sin \theta_{1} \sin \theta_{2}} \sum_{n, m=0}^{N} F_{n, m} \iint_{S} d x d y \cos \left(q_{x} x\right) \frac{\sin \left(\Delta q_{x} x\right)}{x} \frac{\sin \left(\Delta q_{y} y\right)}{y} \times\left[\frac{\left\langle\exp \left[-i q_{z}\left(h_{n}(x, y)-h_{m}(0,0)\right)\right]\right\rangle}{\left\langle\exp \left(-i q_{z} h_{n}\right)\right\rangle\left\langle\exp \left(i q_{z} h_{m}\right\rangle\right.}-1\right] .
$$

Here

$$
F_{n, m}=\Delta \varepsilon_{n} \Delta \varepsilon_{m} \exp \left[-i q_{z}\left(z_{n}-z_{m}\right)\right]\left\langle\exp \left(-i q_{z} h_{n}\right\rangle\left\langle\exp \left(i q_{z} h_{m}\right)\right\rangle,\right.
$$

where $\Theta(q)$ is the Heaviside function. The terms $R_{1}$ and $R_{2}$ describe the contributions of specular and diffuse scattering, respectively. Here the diffuse term $R_{2}$ is caused by the finite size of the slit. The difference of the reflectivities for two widths of the slits may be written in the form

$$
\begin{aligned}
R\left(\Delta \Omega_{1}\right)-R\left(\Delta \Omega_{2}\right)= & \frac{k_{0}^{4}}{(4 \pi)^{2} q_{z}^{2} \sin \theta_{1} \sin \theta_{2}} \sum_{n, m=0}^{N} F_{n m} \iint_{S} d x d y \cos \left(q_{x} x\right) \\
& \times \frac{\sin \left(\Delta q_{x_{1}} x\right) \sin \left(\Delta q_{y_{1}} y\right)-\sin \left(\Delta q_{x_{2}} x\right) \sin \left(\Delta q_{y_{2}} y\right)}{x y}\left[\frac{\left\langle\exp \left[-i q_{z}\left(h_{n}(x, y)-h_{m}(0,0)\right)\right]\right\rangle}{\left\langle\exp \left[-i q_{z} h_{n}\right]\right\rangle\left\langle\exp \left[i q_{z} h_{m}\right]\right\rangle}-1\right] .
\end{aligned}
$$

It is worth to note that this difference depends on the diffuse contribution only and contains the width of the slits. Integration over $x$ and $y$ is restricted by the correlation length $l_{(x y)}$. For most experiments the inequality

$$
\Delta q_{x} l_{(x y)}=\frac{\pi a \sin \theta_{2}}{L} \frac{l_{(x y)}}{\lambda} \ll 1
$$

is valid and we can use the approximation $\sin \left(\Delta q_{x} x\right) \approx \Delta q_{x} x$. Indeed, for the typical experiments we have $a \cong 0.5 \mathrm{~mm}, L$ $\cong 1 \mathrm{~m}, \lambda \cong 0.15 \mathrm{~nm}$, and $\theta_{2} \leq 10^{\circ}$. Therefore the inequality [Eq. (12)] leads to the restriction $l_{(x y)} \ll 500 \mathrm{~nm}$. In this case Eq. (9) can be rewritten in the form

$$
R_{2}=\frac{a k_{0}^{3}}{8 \pi^{2} L q_{z}^{2} \sin \theta_{1}} \sum_{n, m=0}^{N} F_{n, m} \iint_{S} d x d y \cos \left(q_{x} x\right) \frac{\sin \left(\Delta q_{y} y\right)}{y}\left[\frac{\left\langle\exp \left[-i q_{z}\left(h_{n}(x, y)-h_{m}(0,0)\right)\right]\right\rangle}{\left\langle\exp \left[-i q_{z} h_{n}\right]\right\rangle\left\langle\exp \left[i q_{z} h_{m}\right]\right\rangle}-1\right] .
$$

Note that expressions (7), (8), and (13) for the reflectivity do not contain the particular form of the roughness distribution function.

\section{ANALYSIS OF THE EXPERIMENTAL DATA}

For systems with in-plane correlation lengths satisfying the inequality [Eq. (12)], the diffuse contribution to specular

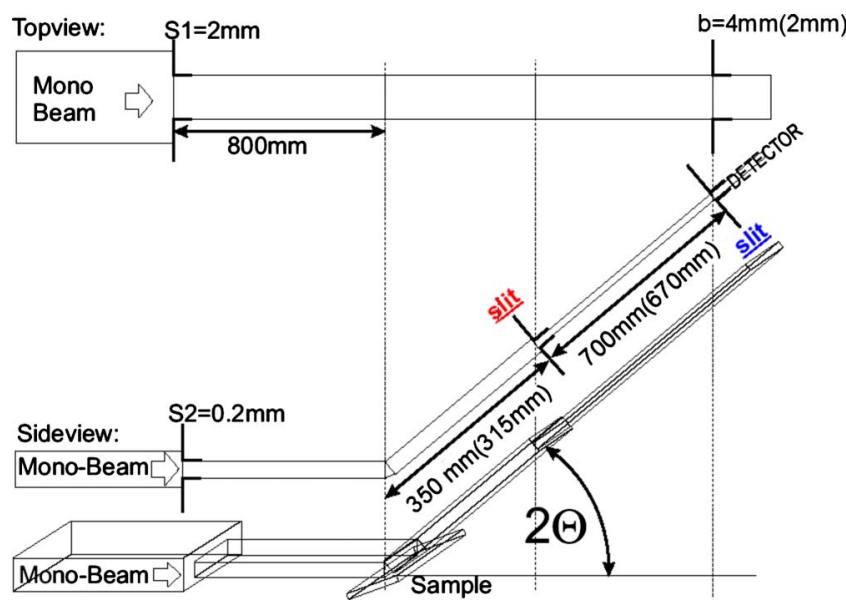

FIG. 1. (Color online) Experimental setup for x-ray scattering investigations. Slit parameters for the sample A are shown without brackets and for sample B with brackets. scattering is proportional to the slit width $a$, independent of the distribution function for the interface roughness. The specular contribution to the scattering $R_{1}$ does not depend on $a$. Therefore the reflectivity $R$ can be written as

$$
R\left(q_{z}, a\right)=R_{1}\left(q_{z}\right)+a I\left(q_{z}\right),
$$

where we used the notation $a I\left(q_{z}\right) \equiv R_{2}\left(q_{z}, a\right)$. Equation (14)

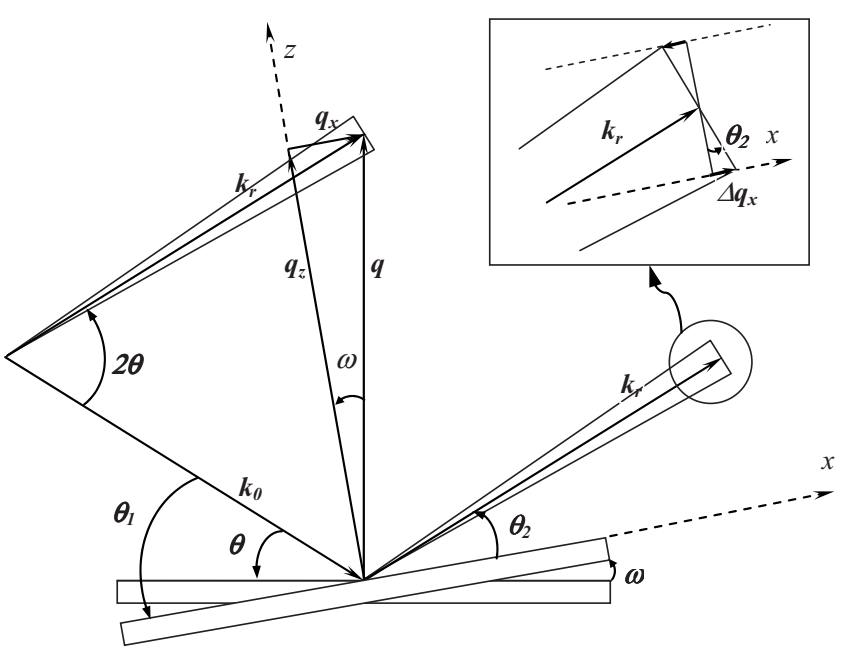

FIG. 2. The geometry of scattering in $\mathbf{q}$ space. The inset shows the wave vectors of scattered radiation which are collected by the detector due to the finiteness of the detector slit size. 


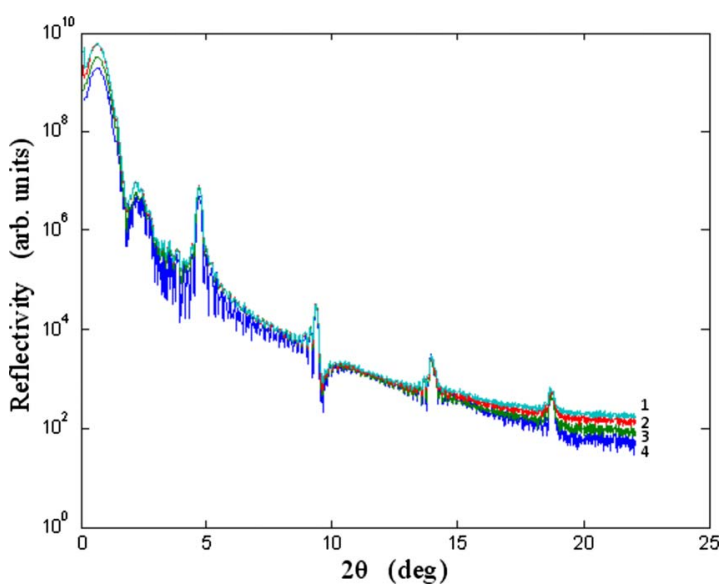

FIG. 3. (Color online) Measured angular dependence of specular $\mathrm{x}$-ray scattering by sample A for various detector slit sizes. From up to down the slit opening angle $\delta=a / L$ equal to: 1 (light blue) -4.29 mrad, 2 (red) $-2.86 \mathrm{mrad}, 3$ (green) $-1.43 \mathrm{mrad}$, and 4 (blue)$0.476 \mathrm{mrad}$.

allows a separation of the angular dependence of the specular scattering $R_{1}\left(q_{z}\right)$ and the diffuse contribution $R_{2}\left(q_{z}, a\right)$ from experimental $\theta_{1}=\theta_{2}$ scans obtained for two different detector slit widths. The equation set for reflectivities $R\left(\theta_{z} ; a_{1}\right)$ and $R\left(\theta_{z} ; a_{2}\right)$ measured for two detector slits of widths $a_{1}$ and $a_{2}$ has the form

$$
\left\{\begin{array}{l}
R\left(q_{z}, a_{1}\right)=R_{1}\left(q_{z}\right)+a_{1} I\left(q_{z}\right) \\
R\left(q_{z}, a_{2}\right)=R_{1}\left(q_{z}\right)+a_{2} I\left(q_{z}\right) .
\end{array}\right.
$$

From this system it is possible to get the inputs $R_{1}$ and $R_{2}$ separately

$$
\left\{\begin{array}{l}
R_{1}\left(q_{z}\right)=\frac{1}{a_{2}-a_{1}}\left[a_{2} R\left(q_{z}, a_{1}\right)-a_{1} R\left(q_{z}, a_{2}\right)\right] \\
R_{2}\left(q_{z}, a_{1}\right)=\frac{a_{1}}{a_{2}-a_{1}}\left[R\left(q_{z}, a_{2}\right)-R\left(q_{z}, a_{1}\right)\right] .
\end{array}\right.
$$

Therefore from experiments with different slit widths the universal function $I\left(q_{z}\right)$ can be found

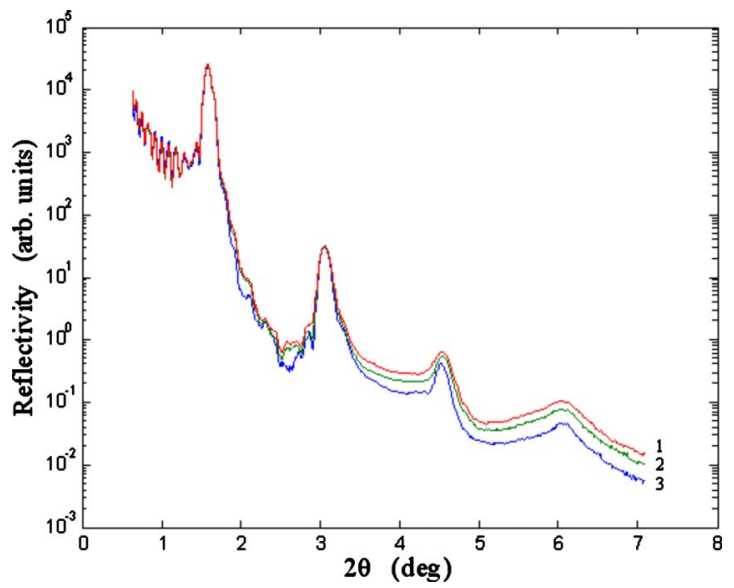

FIG. 4. (Color online) Measured angular dependence of the specular X-ray scattering by sample B for various detector slit sizes. From up to down the slit opening angles $\delta=a / L$ equal to: 1 (red)$4.762 \mathrm{mrad}, 2$ (green) - $3.175 \mathrm{mrad}$, and 3 (blue) $-1.587 \mathrm{mrad}$.

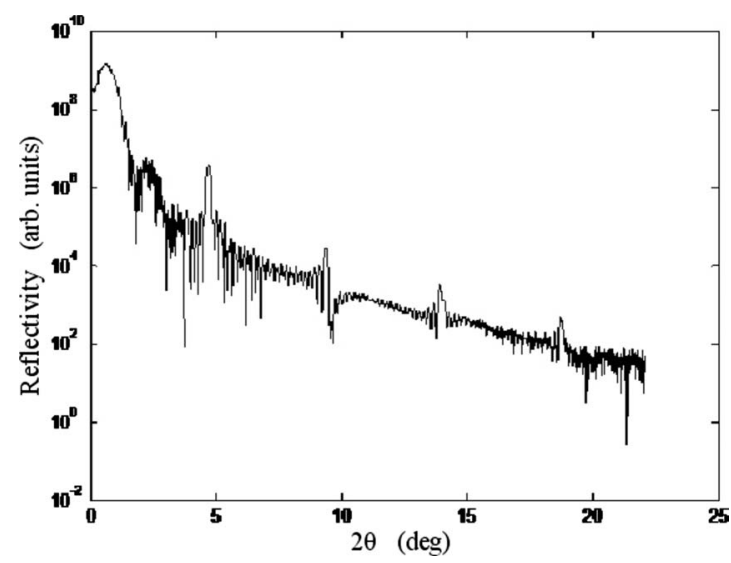

FIG. 5. Angular dependence of the pure specular contribution $R_{1}$ calculated via Eq. (16) from the experimental data presented in Fig. 3.

$$
I\left(q_{z}\right)=\frac{1}{a_{2}-a_{1}}\left[R\left(q_{z}, a_{2}\right)-R\left(q_{z}, a_{1}\right)\right] .
$$

In the experiment we investigated by $\mathrm{x}$-ray scattering two different multilayers:

$[\mathrm{V}(1.212 \mathrm{~nm}) / \mathrm{Fe}(0.715 \mathrm{~nm})]_{25} / \mathrm{V}(24.24 \mathrm{~nm}) / \operatorname{Pd}(5-8 \mathrm{~nm})$ on $\mathrm{MgO}(001)$ substrate (sample $\mathrm{A}$ ) and a multilayer $[\mathrm{CoFeB}(2.55 \mathrm{~nm}) / \mathrm{MgO}(1.8 \mathrm{~nm})]_{15}$ on a $\mathrm{Si} / \mathrm{SiO}_{2}$ substrate (sample B). The subscripts denote the number of repeats in the multilayers. In sample B the alloy was prepared in the proportion $\mathrm{Co}: \mathrm{Fe}: \mathrm{B}=60: 20: 20$. These two multilayer systems were chosen since their interfaces differ significantly. In particular, the interfaces in sample A are considerably smoother than in sample B. The preparation of the multilayers is described in more detail in Refs. 18 and 19. The experimental setup is shown in Fig. 1. The size of the detector slit along the $y$ axis was constant, $b=4 \mathrm{~mm}$ for sample A and $b=2 \mathrm{~mm}$ for sample B. The width of the scattered beam was adjusted by two slits located at distances 350 and $1050 \mathrm{~mm}$ from the specimen for sample A, and at distances 315 and $985 \mathrm{~mm}$ for sample B. The second slit was placed directly in

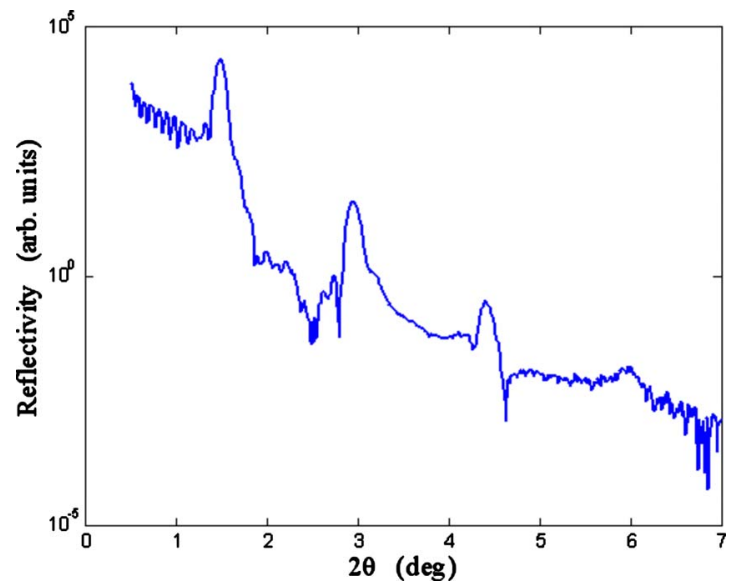

FIG. 6. (Color online) Angular dependence of the pure specular contribution $R_{1}$ calculated via Eq. (16) from the experimental data presented in Fig. 4. 


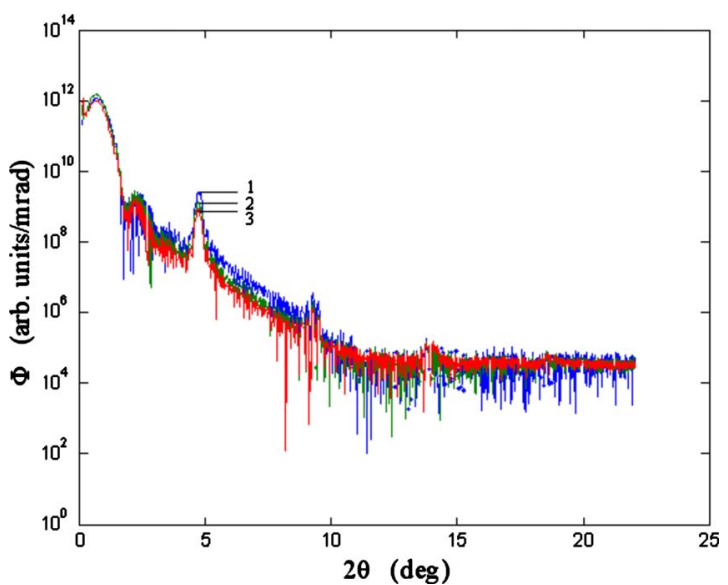

FIG. 7. (Color online) Angular dependence of the normalized function $\Phi$ calculated via Eq. (18) from the experimental data, presented in Fig. 3: 1 (blue) $-\delta_{1}=1.43 \mathrm{mrad}, \delta_{2}=0.476 \mathrm{mrad} ; 2$ (green) $-\delta_{1}=2.86 \mathrm{mrad}, \quad \delta_{2}=0.476 \mathrm{mrad} ; \quad$ and 3 (red) $-\delta_{1}$ $=4.29 \mathrm{mrad}, \delta_{2}=0.476 \mathrm{mrad}$.

front of the detector. The measurements were performed in a series of reflectivity scans in which the resolution was released successively via the detector slit system in the scattering plane. All other parameters were kept constant.

Evidently relations [Eq. (16)] remain valid if we substitute the slit widths $a_{1}$ and $a_{2}$ in the scattering plane by the angles of observation at the receiving detector slits $\delta_{1}$ $=a_{1} / L$ and $\delta_{2}=a_{2} / L$, respectively. All experiments were carried out at the "Hamburg Synchrotron Radiation Laboratory" HASYLAB, Hamburg, Germany, using a six-circle diffractometer at the wiggler beamline W1. For the reflectivity measurements of the samples A and B the monochromator was set to the wavelengths $\lambda=0.154071 \mathrm{~nm}$ and $\lambda$ $=0.11272 \mathrm{~nm}$, respectively. The experimental data are presented in Figs. 3 and 4. Note the curves diverge for large angles of scattering for both systems.

The experimental dependence of the purely specular contribution $R_{1}$ on the scattering angle $2 \theta$ calculated by Eq. (16) are shown in Figs. 5 and 6 . Note that on the log scale the pseudo-Debye-Waller factor determines the decay of $R_{1}$ as const $-\sigma^{2} q_{z}^{2}$, i.e., this decay increases with $2 \theta$. Most of the experimental spectra show a slower decay at large angles. ${ }^{15-17,20-22}$ This means that the contribution of diffuse scattering to the specular spectra have to be taken into account for a proper interpretation of the data.

For sufficiently large scattering angles the difference $R\left(\delta_{1}\right)-R\left(\delta_{2}\right)$ increases proportional to the difference of the slit opening angles $\left(\delta_{1}-\delta_{2}\right)$. The reflectivity differences $R\left(\delta_{1}\right)-R\left(\delta_{2}\right)$ obtained for various slit sizes divided by the $\left(\delta_{1}-\delta_{2}\right)$ is the universal function, which is independent of $\delta_{1}$ and $\delta_{2}$,

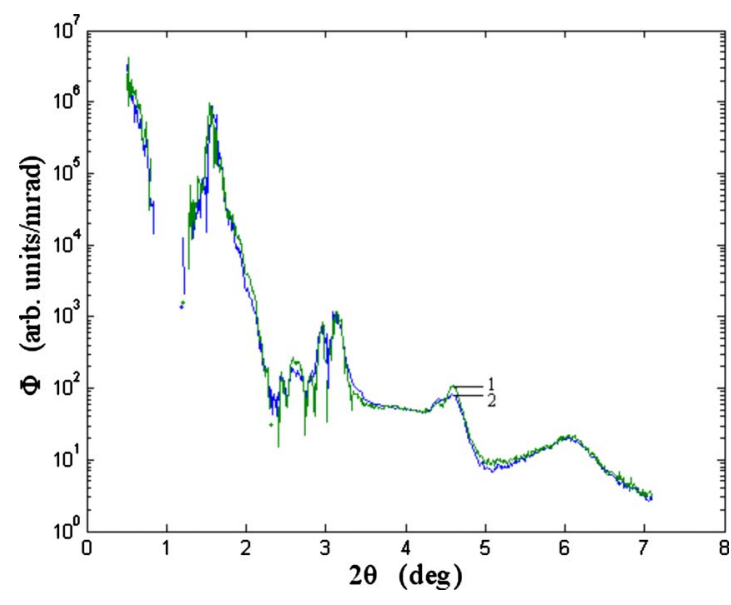

FIG. 8. (Color online) Angular dependence of the normalized function $\Phi$ calculated via Eq. (18) from the experimental data, presented in Fig. 4: 1 (green) $-\delta_{1}=3.175 \mathrm{mrad}, \delta_{2}=1.587 \mathrm{mrad}$ and 2 (blue) $-\delta_{1}=4.762 \mathrm{mrad}, \delta_{2}=1.587 \mathrm{mrad}$.

$$
\Phi=\frac{R\left(\delta_{1}\right)-R\left(\delta_{2}\right)}{\delta_{1}-\delta_{2}}
$$

For both systems the universal function $\Phi$ depending on the scattering angle $2 \theta$ is shown on Figs. 7 and 8 . For large scattering angles these curves indeed coincide independently of the opening angles of the slits $\delta_{1}$ and $\delta_{2}$. The value of the diffuse contribution $R_{2}$ is calculated via Eq. (16) by subtracting the experimental data for various slit openings. As far as for the small scattering angles the contribution of $R_{2}$ is relatively small, its value is determined with rather large error bars in the region of the first two Bragg peaks. Thus the function $\Phi$ is the diffuse contribution to the scattering per unit slit opening angle,

$$
R_{2}\left(q_{z}, a\right)=\delta \Phi=\frac{a}{L} \Phi .
$$

Note that the difference in the interface roughness in these systems results in different angular dependencies for large scattering angles. For sample A the universal function $\Phi$ depends only weakly on the scattering angle and shows only contributions in the neighborhood of Bragg peaks, decaying rapidly with higher orders. For the B sample with rough interfaces this dependence has much more structure and is essentially nonmonotonic. This dependence permits us to obtain information about the correlation properties of the interface roughness.

For a Gaussian distribution of the interface roughness [Eq. (13)] can be transformed as

$$
R_{2}=\frac{a k_{0}^{3}}{8 \pi^{2} L q_{z}^{2} \sin \theta_{1}} \sum_{n, m=0}^{N} F_{n, m} \iint_{S} d x d y \cos \left(q_{x} x\right) \frac{\sin \left(\Delta q_{y} y\right)}{y}\left[\exp \left(q_{z}^{2}\left\langle h_{n}(x, y) h_{m}(0,0)\right\rangle\right)-1\right] .
$$

Expression (10) for $F_{n, m}$ in Eqs. (8) and (20) are replaced in this case by 


$$
F_{n, m}=\Delta \varepsilon_{n} \Delta \varepsilon_{m} \exp \left[-i q_{z}\left(z_{n}-z_{m}\right)\right] \exp \left[-\frac{q_{z}^{2}}{2}\left(\left\langle h_{n}^{2}\right\rangle+\left\langle h_{m}^{2}\right\rangle\right)\right] .
$$

The calculated results of Eqs. (7), (8), (20), and (21) and the experimental data are presented in Figs. 9 and 10. Here we used the standard model for the correlation function of interface roughness. ${ }^{14,15}$ In our calculations the roughness of all interfaces were taken to be the same, besides the interfaces to the substrate and to the cap layers,

$$
\left\langle h_{n}\left(\mathbf{r}_{(x y), 1}\right) h_{m}\left(\mathbf{r}_{(x y), 2}\right)\right\rangle=\sigma_{n} \sigma_{m} \exp \left[-\left(\frac{\left|z_{n}-z_{m}\right|}{l_{z}}\right)^{2}\right] \exp \left[-\left(\frac{\left|\mathbf{r}_{(x y), 1}-\mathbf{r}_{(x y), 2}\right|}{l_{(x y)}}\right)^{2}\right],
$$

where $\sigma_{n}$ is the mean square roughness of the $n$th interface, $l_{n}$ and $l_{(x y)}$ are the correlation lengths transverse and parallel to the interfaces.

\section{MODELS OF THE ROUGHNESS CORRELATION FUNCTIONS}

The interface roughness is characterized by the displacement $h\left(\mathbf{r}_{(x y)}\right)$ along the $z$ axis in the point $\mathbf{r}_{(x y)}$. The probability of the roughness configuration in Gaussian approximation can be written as

$$
P\left[h\left(\mathbf{r}_{(x y)}\right)\right]=\exp \left[-\iint d \mathbf{r}_{(x y), 1} d \mathbf{r}_{(x y), 2} G\left(\mathbf{r}_{(x y), 1}, \mathbf{r}_{(x y), 2}\right) h\left(\mathbf{r}_{(x y), 1}\right) h\left(\mathbf{r}_{(x y), 2}\right)\right],
$$

where the kernel $G\left(\mathbf{r}_{\perp 1}, \mathbf{r}_{\perp 2}\right)$ in general contains the differential operators (see, e.g., Ref. 23). For multilayer films with $N$ interfaces the probability of the displacements of all interfaces may be presented in the form

$$
P\left[h_{1}\left(\mathbf{r}_{(x y)}\right), h_{2}\left(\mathbf{r}_{(x y)}\right), \ldots, h_{N}\left(\mathbf{r}_{(x y)}\right)\right] \sim \exp \left[-\frac{1}{2} \sum_{n, m=1}^{N} \iint d \mathbf{r}_{(x y), 1} d \mathbf{r}_{(x y), 2} G_{n m}\left(\mathbf{r}_{(x y), 1}-\mathbf{r}_{(x y), 2}\right) h_{n}\left(\mathbf{r}_{(x y), 1}\right) h_{m}\left(\mathbf{r}_{(x y), 2}\right)\right]
$$

This form of $P$ assumes that the roughness is homogeneous within each layer. In Fourier representation Eq. (24) gives

$$
P\left(h_{1, \mathbf{q}_{(x y)}} h_{2, \mathbf{q}_{(x y)}} \cdots h_{N, \mathbf{q}_{(x y)}}\right) \sim \exp \left(-\frac{\sqrt{S}}{2} \sum_{n, m=1}^{N} \sum_{\mathbf{q}_{(x y)}} G_{n m, \mathbf{q}_{(x y)}} h_{n, \mathbf{q}_{(x y)}} h_{m, \mathbf{q}_{(x y)}}\right),
$$

where $G_{n m, \mathbf{q}_{(x y)}}$ is the Fourier image of the kernel $G_{n m}\left(\mathbf{r}_{(x y)}\right)$.

$$
h_{\mathbf{q}_{(x y)}}=\frac{1}{\sqrt{S}} \int_{S} d \mathbf{r}_{(x y)} \exp \left(-i \mathbf{q}_{(x y)} \mathbf{r}_{(x y)}\right) h\left(\mathbf{r}_{(x y)}\right), \quad h\left(\mathbf{r}_{(x y)}\right)=\frac{1}{\sqrt{S}} \sum_{\mathbf{q}_{(x y)}} \exp \left(i \mathbf{q}_{(x y)} \mathbf{r}_{(x y)}\right) h_{\mathbf{q}_{(x y)}} .
$$

The probability of the roughness configuration has to obey the normalization condition. This condition is satisfied when the matrix $G_{n m, \mathbf{q}_{(x y)}}$ is positive determined. In this case the correlation function $\left\langle h_{n, \mathbf{q}_{(x y)}} h_{m,-\mathbf{q}_{(x y)}}\right\rangle$ is expressed via elements of the inverse matrix $G^{-1}$,

$$
\left\langle h_{n, \mathbf{q}_{(x y)}} h_{m,-\mathbf{q}_{(x y)}}\right\rangle=\frac{1}{\sqrt{S}}\left(G^{-1}\right)_{n m, \mathbf{q}_{(x y)}} .
$$

In this work we analyze the susceptibility of the x-ray scattering spectrum to the form of the interface roughness correlation function $\left\langle h_{n}\left(\mathbf{r}_{1}^{(x y)}\right) h_{m}\left(\mathbf{r}_{2}^{(x y)}\right)\right\rangle$. Most of the models used in practice assume that the spatial correlation function for multilayer systems decay monotonically in the direction transverse to the layers. In the plane of the layers the weakening of the correlations with the distance can be either monotonic or oscillatory. Oscillations may be caused, for example, by regular distortions of the interface surface or island formation during epitaxial growth. The only severe constrain in case of homogeneous roughness is the positively definiteness of the $G_{n m, \mathbf{q}_{(x y)}}$ matrix. The standard model which describes the monotonic weakening of the roughness correlation with the distance has the form, Eq. (22). We will consider the simple generalization of Eq. (22) which may lead to an oscillation of the correlation function 


$$
\left\langle h_{n}\left(\mathbf{r}_{\perp 1}\right) h_{m}\left(\mathbf{r}_{\perp 2}\right)\right\rangle=\sigma_{n} \sigma_{m} \exp \left[-\left(\frac{\left|z_{n}-z_{m}\right|}{l_{z}}\right)^{2}\right] \exp \left[-\left(\frac{\left|\mathbf{r}_{(x y), 1}-\mathbf{r}_{(x y), 2}\right|}{l_{(x y)}}\right)^{2}\right] J_{0}\left(\frac{\left|\mathbf{r}_{(x y), 1}-\mathbf{r}_{(x y), 2}\right|}{l}\right),
$$

where $J_{0}$ is the zero-order Bessel function. In the limit $l \rightarrow \infty$ Eq. (28) transfers to Eq. (22).

The Fourier transform of the correlation function has the form

$$
\begin{gathered}
\left\langle h_{n, \mathbf{q}_{(x y)}} h_{m,-\mathbf{q}_{(x y)}}\right\rangle=\int d \mathbf{r}_{(x y)} \exp \left(-i \mathbf{q}_{(x y)} \mathbf{r}_{(x y)}\right)\left\langle h_{n}\left(\mathbf{r}_{(x y)}\right) h_{m}(0)\right\rangle \\
=\sigma_{n} \sigma_{m} \exp \left[-\left(\frac{\left|z_{n}-z_{m}\right|}{l_{z}}\right)^{2}\right] \pi l_{(x y)}^{2} \exp \left(-\frac{l_{(x y)}^{2}}{4 l^{2}}\left(1+q_{(x y)}^{2} l^{2}\right)\right) I_{0}\left(\frac{q_{(x y} l_{(x y)}^{2}}{2 l}\right),
\end{gathered}
$$

where $I_{0}(z)$ is the modified Bessel function which admits positive values only. Consequently, although at finite $l$ the correlation function $\left\langle h_{n}\left(\mathbf{r}_{(x y), 1}\right) h_{m}\left(\mathbf{r}_{(x y), 2}\right)\right\rangle$ oscillates, the matrix $G_{\left.n m, \mathbf{q}_{(x)}\right)}$ is positive defined.

Figures 9 and 10 show the results of calculations by Eqs. (7), (8), (20), and (21) and the results obtained from experiments with sample B and described in Sec. III. In these calculations the model for the correlation function with monotonic decay in the plane of the interface [Eq. (22)] was used. The film parameters were chosen so that the angular dependence of the calculated total specular scattering shown in Fig. 9 and diffuse contribution presented in Fig. 10 coincide as much as possible. Figures 11 and 12 illustrate similar calculations for a nonmonotonic correlation function, Eq. (29). The roughness amplitudes of the interfaces were the same as for the model, Eq. (22). The obtained results show that both models, Eqs. (22) and (28), describe the results of the experiments with various width of the detector slit approximately with the same accuracy. The correlation length $l_{(x y)}$ in the model [Eq. (22)] with monotonic decay of the roughness

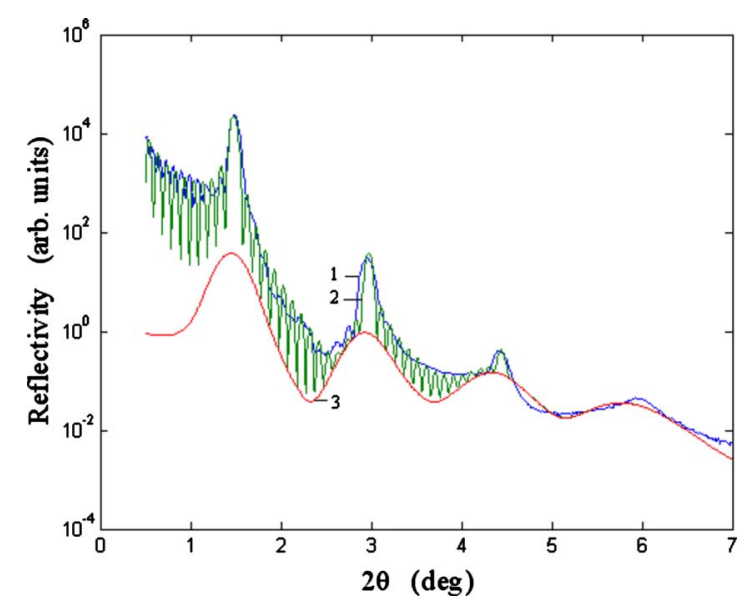

FIG. 9. (Color online) Calculated and measured angular dependence of specular x-ray scattering by sample B: 1 (blue)—measured with $\delta=1.587 \mathrm{mrad} ; 2$ (green)—calculated via Eqs. (7), (8), and (20)-(22); and 3 (red)_diffuse part calculated via Eqs. (20)-(22). For calculations the following set of parameters was used: for substrate: $\sigma=0.5 \mathrm{~nm}$; for multilayer: $\sigma=0.62 \mathrm{~nm}, l_{z}=11 \mathrm{~nm}, l_{(x y)}$ $=25 \mathrm{~nm}$, and $\delta=1.587 \mathrm{mrad}$. correlation is on the order of the parameter $2 l$, which determine the period of the oscillations in the model, Eq. (28). This model also predicts a very large value $l_{(x y)}$, i.e., the correlation function very slowly decays with distance in the interface plane. Both correlation functions, Eqs. (22) and (28), are shown in Fig. 13. Although these correlation functions differ significantly, they lead to very similar effects on x-ray spectra (Figs. 9-12). The x-ray spectra depend only on those regions where the correlations are large and are not sensitive to the tail of the correlation functions. Since both models describe the experiment with similar accuracy we have to conclude that weak long-range roughness correlations cannot be extracted from specular $\mathrm{x}$-ray spectra.

A similar analysis of the $\mathrm{x}$-ray scattering data from sample A leads to the following set of parameters: for substrate and multilayer $-\sigma=0.17 \mathrm{~nm}, l_{z}=0 \mathrm{~nm}, l_{(x y)}=35 \mathrm{~nm}$, and $\delta=1.43 \mathrm{mrad}$; for cap layers: thickness of $\mathrm{V}$ layer is $24.24 \mathrm{~nm} ; \sigma=0.17 \mathrm{~nm}$; for Pd cap layer thickness is $5 \mathrm{~nm}$; $\sigma=0.8 \mathrm{~nm}$.

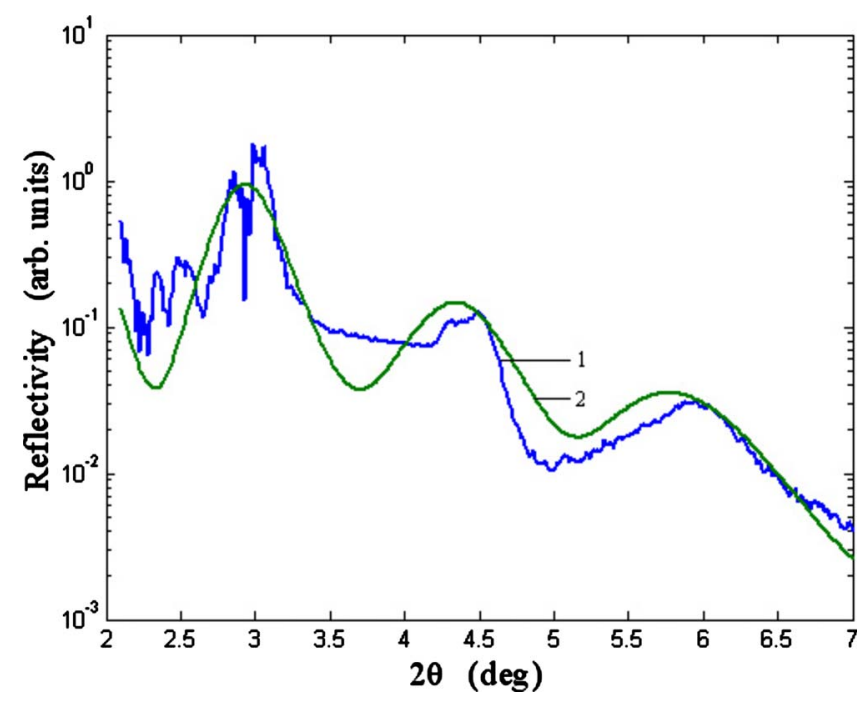

FIG. 10. (Color online) The angular dependence of diffuse contribution to specular x-ray scattering: 1 (blue)—diffuse part $R_{2}$ calculated from the experimental data with the help of universal function $\Phi$ via Eq. (19) and 2 (green)—diffuse part $R_{2}$ calculated via Eqs. (20)-(22). The set of parameters is the same as in caption to Fig. 9. 


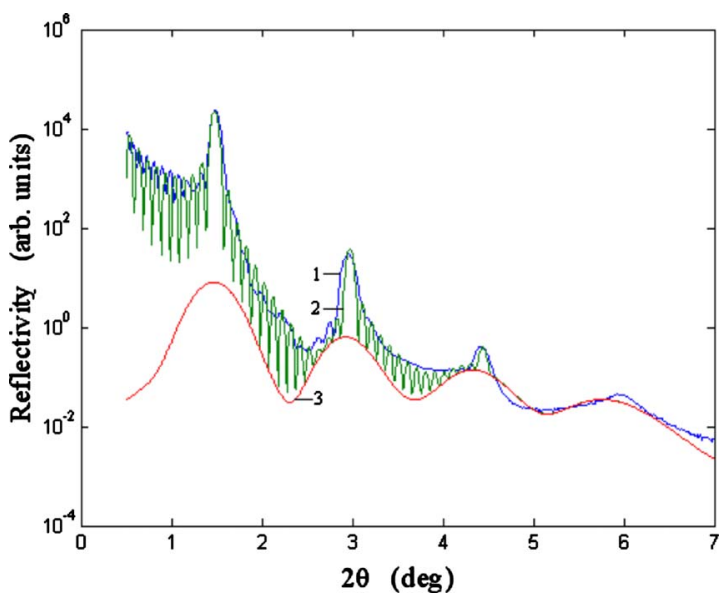

FIG. 11. (Color online) Calculated and measured angular dependence of specular x-ray scattering by sample B: 1 (blue)—measured with $\delta=1.587 \mathrm{mrad} ; 2$ (green)—calculated via Eqs. (7), (8), (20), (21), and (28); and 3 (red)_diffuse part calculated via Eqs. (20), (21), and (28). For calculations the following set of parameters were used: $\sigma=0.5 \mathrm{~nm}$, for substrate and $\sigma=0.62 \mathrm{~nm}, l_{z}=11 \mathrm{~nm}, l_{(x y)}$ $=100 \mathrm{~nm}, l=13 \mathrm{~nm}$, and $\delta=1.587 \mathrm{mrad}$ for multilayer.

\section{DISCUSSION AND SUMMARY}

All experimental $\mathrm{x}$-ray scans are recorded with finite slit openings in front of the detector. Consequently, they contain both specular and diffuse contributions. The measurement procedure using different slit openings for specular $\theta: 2 \theta$ scans as proposed above allows separating both contributions. This opens possibilities for investigating of interface roughness in multilayer systems. It is worth to note that in the literature procedures are described that intend to separate diffuse contributions from specular scans: Savage et al. ${ }^{24}$ and Phang et al. ${ }^{25}$ propose to subtract the diffuse intensity from specular scans by estimating the diffuse intensity from rocking scans near Bragg peaks. Indeed these spectra contain

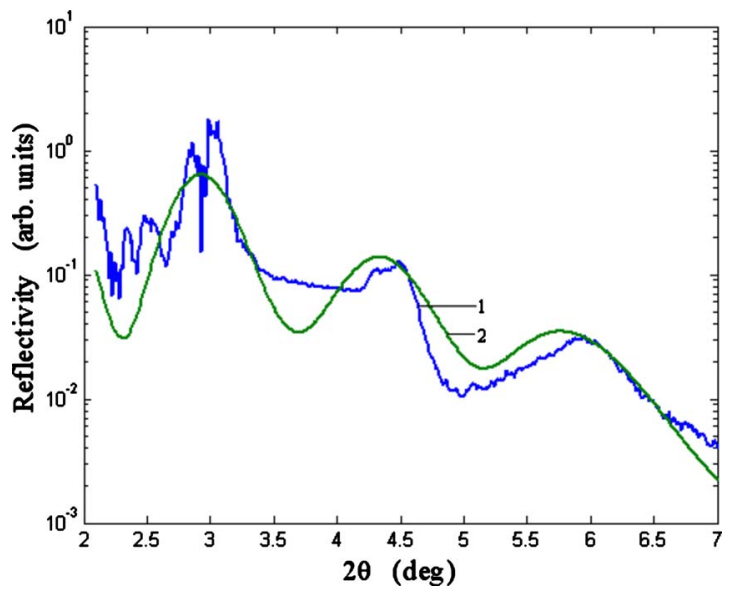

FIG. 12. (Color online) The angular dependence of diffuse contribution to the specular x-ray scattering: 1 (blue) — diffuse part $R_{2}$ calculated from the experimental data with the help of universal function $\Phi$ via Eq. (19), 2 (green)—diffuse part $R_{2}$ calculated via Eqs. (20), (21), and (28). The set of parameters is the same as in caption to Fig. 11.

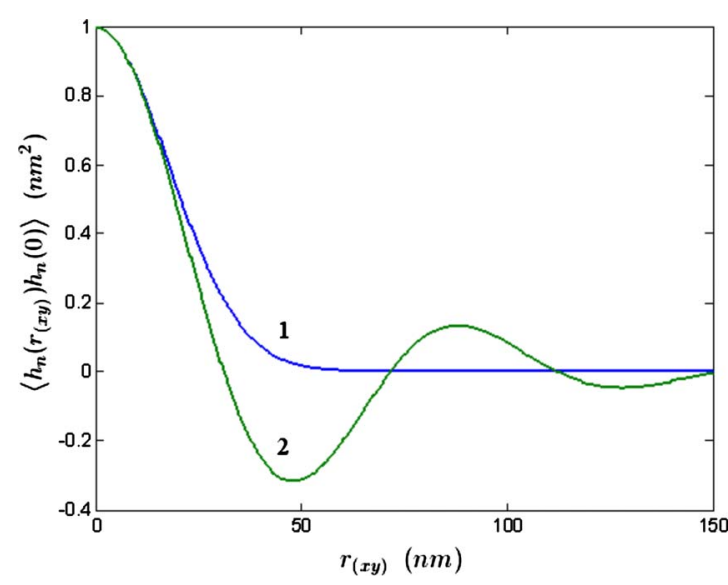

FIG. 13. (Color online) Best-fitting in-plane correlation functions used in calculations. Blue-correlation function defined via Eq. (22) and green-defined via Eq. (28).

instrument limited Bragg peak intensities above a slowly varying diffuse background. However this diffuse contribution has to be measured not only near Bragg peaks but also for all values of $q_{z}$. Comparison of experimental and fitted spectra reported in Refs. 24 and 25 shows a rather bad agreement at large scattering angles. The experimental intensity decreases much more slowly than predicted by the theory. In Ref. 24 such discrepancy was attributed to changing x-ray penetration depth at small glancing angles of incidence or to the violation of a Gaussian distribution of interfacial heights. However, more likely the discrepancy is a consequence of a nonaccurate subtraction of the diffuse background. Salditt et $a l .{ }^{11,12}$ suggested an out-of-plane scattering geometry to determine the diffuse contribution in x-ray scans. This allows for given value of $q_{z}$ to measure the diffuse intensity over a wide range of $q_{y}$. But again if we try to separate pure specular spectra from the diffuse scattering, we need to make such measurement for a large number of $q_{z}$ points. Our experimental approach with different slit openings is simpler, faster, and avoids the experimental problem of how to accurately determine the diffuse intensity over a large area in reciprocal space. In this way specular and diffuse scattering contributions are obtained simultaneously from the same experimental data and do not need to be separated in order to obtain comprehensive information on interface roughness parameters and correlation lengths.

Let us first consider the information that can be extracted from a pure specular spectrum. For any interface roughness this spectrum does not depend neither on in-plane nor on out-of-plane correlation lengths at least in the kinematic approximation, see Eqs. (8) and (10). The decay of scattered intensity is determined solely by the root-mean-square (rms) roughness $\sigma$. Often the interface roughness in multilayers contains a gradient from the substrate toward the surface. Roughness gradients lead to distortions of the phase relationship in the specular spectrum. As a result high-order Bragg peaks can be smoothed out whereas intermediate peaks may disappear. The calculated dependence of specular spectra on roughness gradients is shown in Fig. 14. Similar behavior was simulated in Ref. 26 for Mo/Ni multilayers with a linear variation in the rms roughness. Thus from the pure specular 


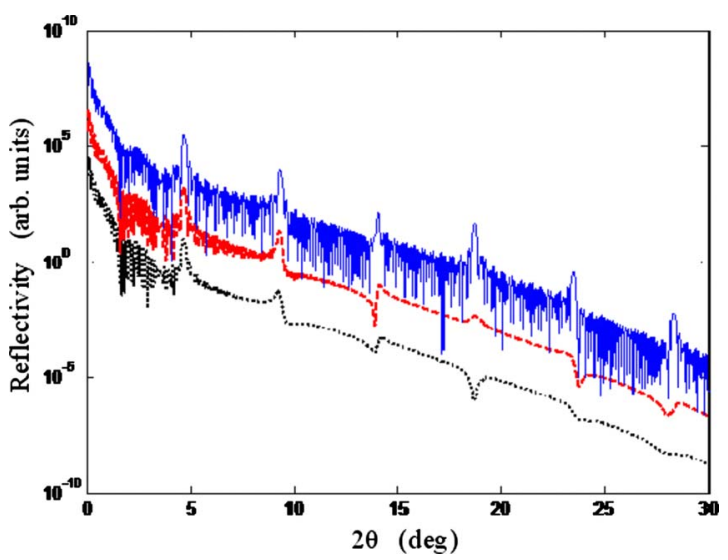

FIG. 14. (Color online) Pure specular x-ray scattering calculated via Eqs. (8) and (21) for the model system $[\mathrm{V}(1.2 \mathrm{~nm}) / \mathrm{Fe}(0.69 \mathrm{~nm})]_{25} / \mathrm{V}(24.24 \mathrm{~nm}) / \mathrm{Pd}(5 \mathrm{~nm})$ on $\mathrm{MgO}$ substrate. For dotted line (black)—rms roughness linearly increase from $\sigma_{0}=0.17 \mathrm{~nm}$ at the interface to the substrate to $\sigma_{N}=3$ $\times 0.17 \mathrm{~nm}$ for the top layer; for dashed line (red)—rms roughness linearly increase from $\sigma_{0}=0.17 \mathrm{~nm}$ at the interface to the substrate to $\sigma_{N}=2 \times 0.17 \mathrm{~nm}$ for the top layer; for solid line (blue) $-\sigma_{n}$ $=0.17 \mathrm{~nm}$ is constant for all interfaces. The dashed and dotted curves were shifted along $Y$ axes for clarity.

part of x-ray scattering it is possible to estimate the rms roughness amplitude and its variation from layer to layer. Experimental control over the interface roughness can be achieved via the choice of substrate, the deposition temperature, the growth rate, and the interaction with inert gases during the sputtering process. Examples of roughness variation in sputtered $\mathrm{Nb} / \mathrm{Al}_{2} \mathrm{O}_{3}$ and $\mathrm{W} / \mathrm{Si}$ multilayers in $\mathrm{Ar}$ atmosphere are presented in Refs. 12 and 21, respectively. It was found that the rms roughness increases drastically when the Ar pressure is raised above a critical value. In these samples the disappearance of high-order Bragg peaks in $\mathrm{x}$-ray spectra was explained by a cumulative roughness model. ${ }^{24,26}$ However, it was noticed that the intensity of the specular scattering for samples with large rms roughness decayed slower than predicted by theory. ${ }^{12}$ This is most likely due to the diffuse contribution in the specular spectra. If the diffuse component exceeds the specular one for a given slit width, then it also leads to a suppression of higher order Bragg peaks.

Now we will turn our discussion to the dependence of the pure diffuse scattering $R_{2}$ on roughness correlations. The importance of the diffuse scattering in specular x-ray spectra was emphasized in Ref. 26. The authors claim that with moderately rough interfaces the diffuse component constitutes the majority of experimental spectra and that its fine structure would provide the main information about interface roughness. However, diffuse spectra depend on the in-plane correlation length $l_{(x y)}$ and the out-of-plane correlation length $l_{z}$ in different ways. If $R_{2}$ contains well-defined lines (streaks) corresponding to the Bragg peaks then $l_{z}$ of the system is large. Small $l_{z}$ values as compared to the multilayer period $d$ will lead to a suppression of these peaks. Such behavior is illustrated in Fig. 15. The value $R_{2}\left(q_{z}\right)$ turns out

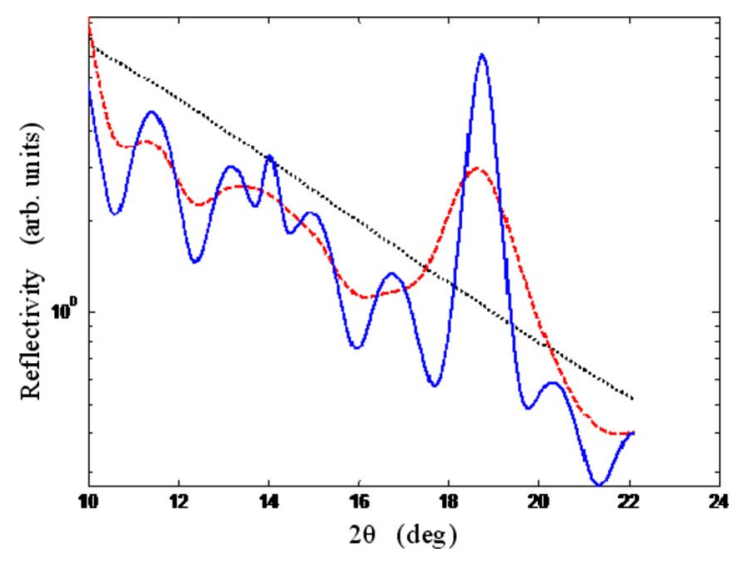

FIG. 15. (Color online) The diffuse contribution to the specular $\mathrm{X}$-ray scattering calculated via Eqs. (20)-(22) for model system $[V(1.2 \mathrm{~nm}) / \mathrm{Fe}(0.69 \mathrm{~nm})]_{25} / \mathrm{V}(24.24 \mathrm{~nm}) / \mathrm{Pd}(5 \mathrm{~nm})$ on $\mathrm{MgO}$ substrate. The following set of parameters was used: $\sigma_{n}=0.17 \mathrm{~nm}$ for all interfaces, $l_{(x y)}=10 \mathrm{~nm}$. For dotted line (black) $-l_{z}=0 \mathrm{~nm}$, for dashed line (red) $-l_{z}=5 \mathrm{~nm}$, and for solid line (blue) $-l_{z}$ $=15 \mathrm{~nm}$.

to be insensitive to the type of in-plane roughness correlation function-Eq. (22) or Eq. (28). Figure 16 shows that the in-plane correlation length $l_{(x y)}$ affects only the magnitude of
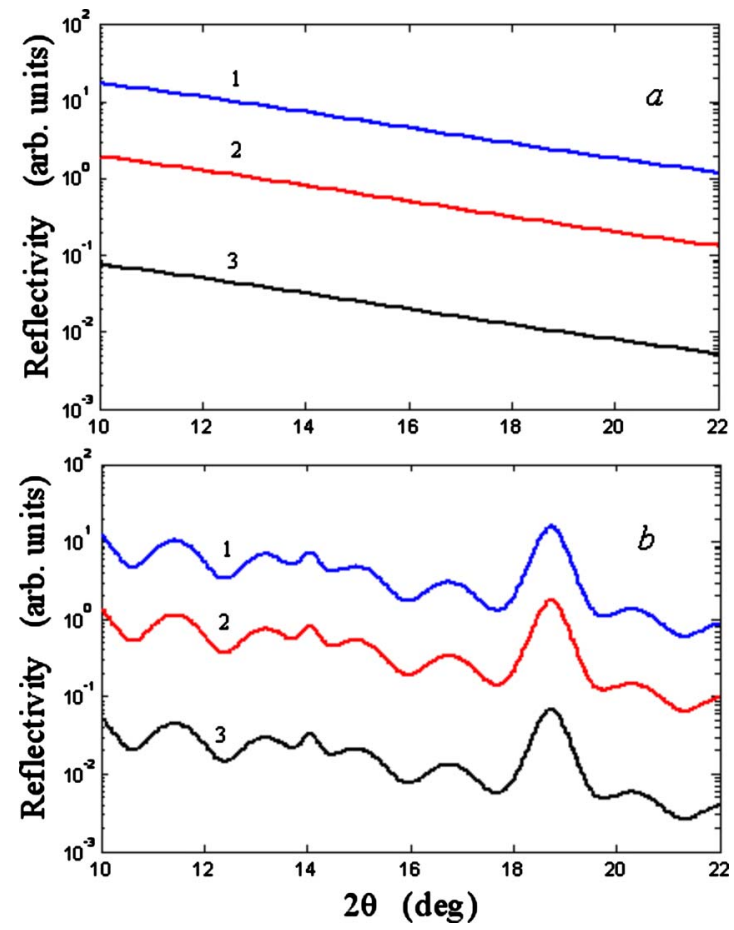

FIG. 16. (Color online) The diffuse contribution to the specular X-ray scattering calculated via Eqs. (20)-(22) for the same model system as in Fig. 15. (a) The following set of parameters was used: $\sigma_{n}=0.17 \mathrm{~nm}, l_{z}=0 \mathrm{~nm}$ for all interfaces. For 1 (blue) line $l_{(x y)}$ $=15 \mathrm{~nm}$, for 2 (red) line $l_{(x y)}=5 \mathrm{~nm}$, and for 3 (black) line $l_{(x y)}$ $=1 \mathrm{~nm}$. (b) The following set of parameters was used: $\sigma_{n}$ $=0.17 \mathrm{~nm}, l_{z}=15 \mathrm{~nm}$ for all interfaces. For 1 (blue) line $l_{(x y)}$ $=15 \mathrm{~nm}$, for 2 (red) line $l_{(x y)}=5 \mathrm{~nm}$, and for 3 (black) line $l_{(x y)}$ $=1 \mathrm{~nm}$. 
the diffuse contribution but does not change the general shape of the $R_{2}(\theta)$ dependence. As expected, the diffuse contribution increases with increasing $l_{(x y)}$.

As already mentioned, during the growth of multilayers the roughness of interfaces often develops a gradient from the substrate to the surface. A theoretical model assuming a linear change in the rms roughness was reported in Ref. 26 on the basis of a Taylor expansion of the reflectivity amplitude of the multilayer. In multilayer systems the variation in the mean square roughness $\sigma_{n}$ with layer number $n$ affects both, the specular and the diffuse contributions. In particular, as shown in Fig. 17, for large correlation lengths $l_{z}$ the linear increase in the interface roughness amplitude leads to a decrease and broadening of peaks in the diffuse contribution to the specular spectrum. Such type of angular dependence of the diffuse contribution to the specular spectra of multilayers was also pointed out in Ref. 26.

In summary, we propose a measurement protocol to determine simultaneously both the specular and the diffuse part of $\mathrm{x}$-ray reflectivity intensities from thin films and multilayers. This protocol requires to take the same scan several times with different slit openings for the detector. From fitting the data the roughness parameters and the correlation lengths are obtained. It should be noted that the specular part yields the rms roughness parameter $\sigma$, whereas from the diffuse intensity the in- and out-of-plane correlation lengths are extracted. The amplitude of the diffuse intensity is also damped by the rms roughness. However, the main information to be gained from the diffuse scattering is not the roughness but the structural correlation functions. In the future this method may be extended to magnetic correlation functions.

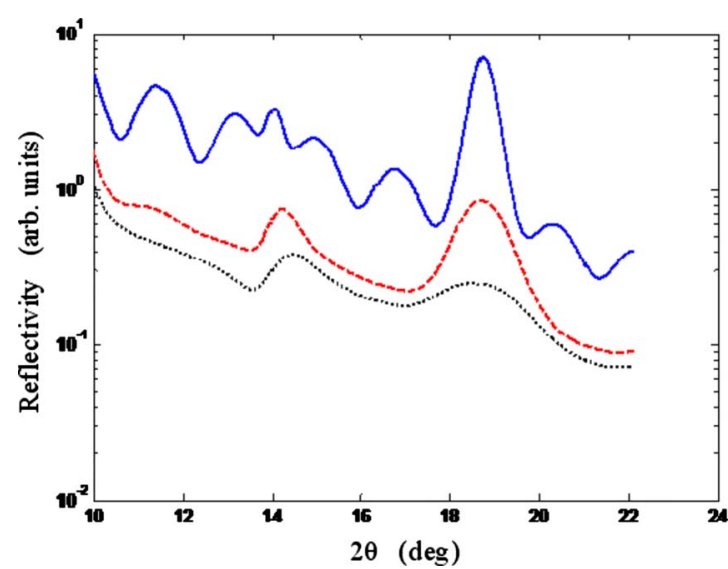

FIG. 17. (Color online) Diffuse contribution to the specular X-ray scattering calculated via Eqs. (20)-(22) for the same model system as in Fig. 15. The following set of parameters was used: $l_{z}$ $=15 \mathrm{~nm}, l_{(x y)}=10 \mathrm{~nm}$. For dotted line (black): rms roughness linearly increases from $\sigma_{0}=0.17 \mathrm{~nm}$ at the interface to the substrate to $\sigma_{N}=6 \times 0.17 \mathrm{~nm}$ for the top layer. For dashed line (red): $\mathrm{rms}$ roughness linearly increases from $\sigma_{0}=0.17 \mathrm{~nm}$ at the interface to the substrate to $\sigma_{N}=3 \times 0.17 \mathrm{~nm}$ for the top layer. For solid line (blue): rms roughness $\sigma_{n}=0.17 \mathrm{~nm}$ constant for all interfaces.

\section{ACKNOWLEDGMENTS}

The authors are grateful to M. Vadala for allowing them to use her sample for the scans shown in Fig. 4 and to W. Caliebe for support at the W1 beamline at the HASYLAB. This work was partly supported by cooperative RFBR-DFG under Grants No. RFBR10-02-913300 and No. DFG ZA 161/ 19.
${ }^{1}$ S. D. Bader, Rev. Mod. Phys. 78, 1 (2006).

${ }^{2}$ S. D. Bader, K. S. Buchanan, S.-H. Chung, K. Yu Guslienko, A. Hoffmann, Y. Ji, and V. Novosad, Superlattices Microstruct. 41, 72 (2007).

${ }^{3}$ P. Skomski, J. Phys.: Condens. Matter 15, R841 (2003).

${ }^{4}$ S. A. Wolf, D. D. Awschalom, R. A. Buhrman, J. M. Daughton, S. von Molna, M. L. Roukes, A. Y. Chtchelkanova, and D. M. Treger, Science 294, 1488 (2001).

${ }^{5}$ J. C. Slonczewski, J. Magn. Magn. Mater. 126, 374 (1993).

${ }^{6}$ A. Schreyer, J. F. Ankner, Th. Zeidler, H. Zabel, M. Schäfer, J. A. Wolf, P. Grünberg, and C. F. Majkrzak, Phys. Rev. B 52, 16066 (1995).

${ }^{7}$ W. Kuch, L. I. Chelaru, F. Offi, J. Wang, M. Kotsugi, and J. Kirschner, Nature Mater. 5, 128 (2006).

${ }^{8}$ J. F. Gregg, I. Petej, E. Jouguelet, and C. Dennis, J. Phys. D: Appl. Phys. 35, R121 (2002).

${ }^{9}$ U. Pietsch, V. Holy, and T. Baumbach, High-Resolution X-Ray Scattering (Springer-Verlag, Berlin, New York, 2004).

${ }^{10} \mathrm{G}$. Renaud, R. Lazzari, and F. Leroy, Surf. Sci. Rep. 64, 255 (2009).

${ }^{11}$ T. Salditt, T. H. Metzger, J. Peisl, and X. Jiang, J. Phys. III 4, 1573 (1994)

${ }^{12}$ T. Salditt, T. H. Metzger, J. Peisl, Ch. Morawe, and H. Zabel, in Evolution of Thin-Film and Surface Structure and Morphology, edited by B. G. Demczyk et al., MRS Symposia Proceedings No. 355 (Materials Research Society, Pittsburgh, 1995), p. 269.

${ }^{13}$ F. Salah, B. Harzallah, and A. van der Lee, J. Appl. Crystallogr. 40, 813 (2007).

${ }^{14}$ S. K. Sinha, J. Phys. III 4, 1543 (1994).

${ }^{15}$ S. K. Sinha, E. B. Sirota, S. Garoff, and H. B. Stanley, Phys. Rev. B 38, 2297 (1988).

${ }^{16}$ J. P. Schlomka, M. Tolan, L. Schwalowsky, O. H. Seeck, J. Stettner, and W. Press, Phys. Rev. B 51, 2311 (1995).

${ }^{17}$ V. P. Romanov, S. V. Ulyanov, V. M. Uzdin, G. Nowak, M. Vadala, and H. Zabel, J. Phys. D 41, 115401 (2008).

${ }^{18}$ G. Nowak, H. Zabel, K. Westerholt, I. Garifullin, M. Marcellini, A. Liebig, and B. Hjörvarsson, Phys. Rev. B 78, 134520 (2008).

${ }^{19}$ M. Vadalá, K. Zhernenkov, M. Wolff, B. P. Toperverg, K. Westerholt, H. Zabel, P. Wisniowski, S. Cardoso, and P. P. Freitas, J. Appl. Phys. 105, 113911 (2009).

${ }^{20}$ D. E. Savage, N. Schimke, Y.-H. Phang, and M. G. Lagally, J. Appl. Phys. 71, 3283 (1992).

${ }^{21}$ T. Salditt, D. Lott, T. H. Metzger, J. Peisl, G. Vignaud, P. Høghøj, O. Schärpf, P. Hinze, and R. Lauer, Phys. Rev. B 54, 5860 (1996).

${ }^{22}$ R. Schad, P. Belien, G. Verbanck, V. V. Moshchalkov, Y. Bruynseraede, H. E. Fischer, S. Lefebvre, and M. Bessiere, Phys. Rev. B 59, 1242 (1999). 
${ }^{23}$ A. Z. Patashinskii, P. J. Shepherd, and V. L. Pokrovskii, Fluctuation Theory of Phase Transitions (Pergamon Press, Oxford, 1995).

${ }^{24}$ D. E. Savage, J. Kleiner, N. Schimke, Y.-H. Phang, T. Jankowski, J. Jacobs, R. Kariotis, and M. G. Lagally, J. Appl. Phys.
69, $1411(1991)$

${ }^{25}$ Y. H. Phang, D. E. Savage, R. Kariotis, and M. G. Lagally, J. Appl. Phys. 74, 3181 (1993).

${ }^{26}$ A. P. Payne and B. M. Clemens, Phys. Rev. B 47, 2289 (1993). 